\title{
Identification and characterization of uncoupling protein 4 in fat body and muscle mitochondria from the cockroach Gromphadorhina cocquereliana
}

\author{
Malgorzata Slocinska • Nina Antos-Krzeminska • \\ Grzegorz Rosinski • Wieslawa Jarmuszkiewicz
}

Received: 28 June 2011 / Accepted: 13 September 2011 / Published online: 14 October 2011

(C) The Author(s) 2011. This article is published with open access at Springerlink.com

\begin{abstract}
We have identified and characterized an uncoupling protein in mitochondria isolated from leg muscle and from fat body, an insect analogue tissue of mammalian liver and adipose tissue, of the cockroach Gromphadorhina coquereliana (GcUCP). This is the first functional characterization of UCP activity in isolated insect mitochondria. Bioenergetic studies clearly indicate UCP function in both insect tissues. In resting (non-phosphorylating) mitochondria, cockroach GcUCP activity was stimulated by the addition of micromolar concentrations of palmitic acid and inhibited by the purine nucleotide GTP. Moreover, in phosphorylating mitochondria, GcUCP activity was able to divert energy from oxidative phosphorylation. Functional studies indicate a higher activity of GcUCP-mediated uncoupling in cockroach muscle mitochondria compared to fat body mitochondria. GcUCP activation by palmitic acid resulted in a decrease in superoxide anion production, suggesting that protection against mitochondrial oxidative stress may be a physiological role of UCPs in insects. GcUCP protein was immunodetected using antibodies raised against human UCP4 as a single band of around $36 \mathrm{kDa}$. GcUCP protein expression in cockroach muscle mitochondria was significantly higher compared to mitochondria isolated from fat body. LC-MS/MS analyses revealed $100 \%$ sequence identities for peptides obtained
\end{abstract}

M. Slocinska $(\square) \cdot$ G. Rosinski

Department of Animal Physiology and Development,

Adam Mickiewicz University,

Poznan, Poland

e-mail: slocina@amu.edu.pl

N. Antos-Krzeminska • W. Jarmuszkiewicz $(\bowtie)$

Department of Bioenergetics, Adam Mickiewicz University,

Poznan, Poland

e-mail: wiesiaj@amu.edu.pl from GcUCP to UCP4 isoforms from D. melanogaster (the highest homology), human, rat or other insect mitochondria. Therefore, it can be proposed that cockroach GcUCP corresponds to the UCP4 isoforms of other animals.

Keywords Insects $\cdot$ Fat body $\cdot$ Mitochondria $\cdot$ Muscle . Uncoupling protein

\begin{tabular}{|c|c|}
\hline \multicolumn{2}{|c|}{ Abbreviations } \\
\hline BSA & bovine serum albumin \\
\hline DmUCP & uncoupling protein of Drosophila melanogaster \\
\hline GcUCP & $\begin{array}{l}\text { uncoupling protein of Gromphadorhina } \\
\text { coquereliana }\end{array}$ \\
\hline $\mathrm{O}_{2}^{--}$ & superoxide anion \\
\hline PA & palmitic acid \\
\hline RCR & respiratory control ratio \\
\hline ROS & reactive oxygen species \\
\hline $\mathrm{UCP}$ & uncoupling protein \\
\hline
\end{tabular}

\section{Introduction}

Uncoupling proteins (UCPs) are integral proteins of the inner mitochondrial membrane that belong to the large family of mitochondrial anion carriers. UCPs catalyze proton leaking into the matrix and dissipate the proton electrochemical gradient built up during electron transport through the mitochondrial respiratory chain (for a review, see Cannon et al. 2006; Echtay 2007; Sluse et al. 2006; Nicholls 2006). A direct consequence of the UCP activity stimulated by free fatty acids is a decrease in oxidative phosphorylation efficacy (Skulachev 1996). The existence of a conserved mechanism of energy dissipation through 
UCPs in almost all eukaryotic cells suggests a crucial role for this protein in energy metabolism.

The first described uncoupling protein, UCP1 or thermogenin, which is present in brown adipose tissue acts as a regulator of heat generation in newborn, hibernating and cold-acclimated animals (Nicholls and Locke 1984). UCP1 has also been detected in the thymus where it has the capacity to regulate metabolic flux and the production of reactive oxygen species (ROS) (Carroll et al. 2005). The widespread presence of other UCPs in eukaryotes, nonthermogenic animal tissues (mammals, fish, birds and insects), plants and unicellular organisms implies that these proteins may elicit functions other than thermogenesis (for a review, see Cannon et al. 2006; Echtay 2007; Jarmuszkiewicz et al. 2010; Sluse et al. 2006; Nicholls 2006; Vercesi et al. 2006). However, the physiological functions of UCP1 homologues are still under debate. They can regulate energy metabolism through modulation of the proton electrochemical gradient and ROS production. Because UCP activation decreases ROS production, UCPs are considered an antioxidant system that prevents cellular oxidative damage at the expense of the oxidative phosphorylation yield. Moreover, mammalian UCP2 (ubiquitously expressed in almost all mammalian tissues) and UCP3 (predominantly expressed in skeletal muscle) might be implicated in numerous physiological and pathological phenomena, e.g., body weight regulation, fatty acid metabolism, inflammation, diabetes, and heart failure (Nedergaard et al. 2005; Harper and Gerritis 2004). The brain specific UCPs, UCP4 and UCP5, might play an important role in apoptosis, neuroprotection, neuronal differentiation and synaptic plasticity in the brain (Kim-Han and Dugan 2005).

Some sequence analyses suggest that UCP1, UCP2 and UCP3 on the one hand, and UCP4 and UCP5 (closely related rather to oxoglutarate and dicarboxylate carriers) on the other hand, belong to distinct groups (Borecky et al. 2001; Ledesma et al. 2002). UCP4 and UCP5 share about $35-40 \%$ amino acid identity with UCP1-3. Mammalian UCP4 is exclusively expressed in different regions of the brain and spinal cord (Mao et al. 1999; Smorodchenko et al. 2009). UCP5 expression is highly enriched in the mammalian nervous system and is located exclusively in neurons (Sanchis et al. 1998; Yu et al. 2000; Kim-Han et al. 2001). The evolution of UCP isoforms is under debate. Hanak and Jezek (2001) proposed that UCP4 represents an ancestral UCP form for all UCPs and that UCP1-3 developed later during evolution. Their phylogenetic analysis suggests that invertebrate UCPs, like those of Drosophila melanogaster and Caenorhabditis elegans, are closely related to vertebrate UCP4. Xenopus laevis UCP4 is present in all subdivisions of the brain and has a very high level of expression in peripheral tissues such as liver and kidney (Keller et al. 2005). The peripheral tissue distribution of $X$. laevis UCP4 reinforces the hypothesis that UCP4 might be the ancestral UCP form from which other UCPs diverged (Hanak and Jezek 2001; Keller et al. 2005). On the other hand, a phylogenetic analysis by Sokolova and Sokolov (2005) suggests an early evolutionary divergence of UCPs and at least three clades in invertebrates, closely related to vertebrate UCP1-3, UCP4, and UCP5, respectively.

An amino acid sequence homology analysis revealed the existence of four putative UCPs in D. melanogaster, UCP4a, UCP4b, UCP4c, and UCP5 (Jezek 2002). As the first insect UCP, D. melanogaster UCP5 (DmUCP5) was functionally characterized in the heterologous yeast system where its expression reduced mitochondrial membrane potential and increased the respiration rate (Fridell et al. 2004). Similar to other UCPs, the uncoupling activity of DmUCP5 is augmented by fatty acids and inhibited by purine nucleotides. DmUCP5 is expressed throughout fly development but at a higher level in adults where it is expressed most abundantly in the head. Later studies with DmUCP5 knockout flies indicated the involvement of DmUCP5 in D. melanogaster metabolism and aging (Sanchez-Blanco et al. 2006). DmUCP5 knockout flies were highly sensitive to starvation stress and gained less weight compared to controls on high calorie diets. Moreover, DmUCP5 knockout flies exhibited a decreased level of fertility and lived longer than controls on low calorie diets. They had a lower body composition of sugars (glucose and trehalose), and during starvation, their triglyceride reserves were used up faster compared to controls. These data indicate that DmUCP5 is important for maintaining metabolic homeostasis in the fly and might influence the hormonal control of metabolism (Sanchez-Blanco et al. 2006).

To date, there are no functional studies involving insect UCPs in isolated mitochondria. The functional characterization of $D$. melanogaster UCP in the heterologous yeast system (Fridell et al. 2004) and the loss-of-function investigations using UCP5 knockout flies (Sanchez-Blanco et al. 2006) are the only studies on function and the metabolic importance of UCPs in insects. Thus, the aim of the present study was to identify molecularly and to characterize functionally Gromphadprhina coquereliana UCP (GcUCP). G. coquereliana is a rather large insect, which makes it a convenient and interesting experimental model for bioenergetic studies using mitochondria isolated from two metabolically different tissues. We studied GcUCP in mitochondria isolated from two peripheral cockroach tissues: (a) from fat body, an insect tissue analogous to mammalian liver and adipose tissue, and (b) from leg muscle, a highly metabolic and energy-dependent tissue. 
The discovery of UCP in the cockroach might have implications for better understanding the bioenergetics, metabolism and function of fat body and muscle in insects. Moreover, the partial amino acids sequence identification of GcUCP, which strongly indicates a UCP4 isoform, could shed light on our understanding of the evolution of mitochondrial UCPs.

\section{Materials and methods}

Animals

Cockroaches (Gromphadorhina coquereliana) were reared under laboratory conditions at $28{ }^{\circ} \mathrm{C}$ and $65-70 \%$ relative humidity. Food (lettuce, carrot, powder milk, and eggs) and water were provided ad libitum. Young adult males were selected for use.

\section{Mitochondrial isolation}

G. coquereliana adults were briefly chilled on ice. Afterwards, the leg muscle and fat body were quickly dissected and collected in ice-cold isolation media. They were cleaned of tracheoles and connective tissues. Muscle mitochondria were isolated in a medium containing $100 \mathrm{mM} \mathrm{KCl}, 50 \mathrm{mM}$ Tris- $\mathrm{HCl}$ (pH 7.4), $1 \mathrm{mM}$ $\mathrm{K}_{2} \mathrm{HPO}_{4}$, and $0.2 \%$ bovine serum albumin (BSA). Muscle tissue were chopped into small pieces within a minute and then homogenized in isolation medium with a teflon-glass homogenizer at $1,000 \mathrm{rpm}$ for four strokes. Muscle homogenate was centrifuged at $500 \mathrm{~g}$ for $10 \mathrm{~min}$. The obtained supernatant was centrifuged at $10,000 \mathrm{~g}$ for $10 \mathrm{~min}$. The pellet-containing mitochondria was incubated in 5\% BSA for $10 \mathrm{~min}$ and then washed twice in isolation medium without BSA and centrifuged at $6,000 \mathrm{~g}$ for $10 \mathrm{~min}$. This step was necessary to deplete muscle mitochondria of remaining fatty acids. The final mitochondrial pellet was resuspended in a medium containing $0.2 \mathrm{M}$ mannitol, $0.1 \mathrm{M}$ sucrose, $10 \mathrm{mM}$ Tris- $\mathrm{HCl}$ (pH 7.4), and $0.1 \mathrm{mM}$ EDTA and stored on ice for further assays.

Mitochondria from the fat body tissue were isolated according to a modified procedure (Sujak 1984). Fat bodies were transferred into the isolation medium containing $0.25 \mathrm{M}$ sucrose, $1 \mathrm{mM}$ EDTA, $5 \mathrm{mM}$ Tris- $\mathrm{HCl}$, and $1 \%$ BSA and then homogenized with a teflon-glass homogenizer. Fat body homogenate was centrifuged at $800 \mathrm{~g}$ for $10 \mathrm{~min}$. The obtained supernatant was centrifuged at $12,000 \mathrm{~g}$ for $10 \mathrm{~min}$. The mitochondrial pellet was then washed twice in medium containing 0.25 $\mathrm{M}$ sucrose, $1 \mathrm{mM}$ EDTA, and $5 \mathrm{mM}$ Tris- $\mathrm{HCl}$ and centrifuged at $8,000 \mathrm{~g}$ for $10 \mathrm{~min}$. The final pellet was resuspended in the same medium.
All of the above-mentioned procedures were performed at $4{ }^{\circ} \mathrm{C}$. The presence of BSA in the isolation media allowed for the chelation of free fatty acids from the mitochondrial suspension. Mitochondrial protein concentrations were determined using the Bradford method (Bradford 1976).

\section{Mitochondrial oxygen consumption}

Oxygen consumption of $G$. coquereliana fat body and muscle mitochondria was measured at $25{ }^{\circ} \mathrm{C}$ with a Clarktype electrode (Oxytherm, Hansatech) in $0.8 \mathrm{ml}$ of the incubation medium containing $0.2 \mathrm{M}$ mannitol, $75 \mathrm{mM}$ sucrose, $10 \mathrm{mM} \mathrm{KCl}, 0.1 \mathrm{mM}$ EDTA, $10 \mathrm{mM} \mathrm{K}_{2} \mathrm{HPO}_{4}$, and $10 \mathrm{mM}$ Tris- $\mathrm{HCl}$ (pH 7.4). Succinate $(10 \mathrm{mM})$ or pyruvate $(10 \mathrm{mM})$ plus malate $(10 \mathrm{mM})$ were used as oxidizable substrates for the measurements of fat body and muscle mitochondrial respiration, respectively. These mitochondrial respiratory substrates were the best found for these mitochondria. To exclude the activities of the ATP/ADP antiporter and the ATP synthase, carboxyatractyloside $(1.5 \mu \mathrm{M})$ and oligomycin $\left(1 \mathrm{mg} \times \mathrm{mg}^{-1}\right.$ mitochondrial protein) were used in resting (State 4) measurements. The total amount of oxygen consumed during phosphorylating respiration (State 3) induced by a pulse of ADP (250 nmoles) was used for calculations of the ADP/O ratio. Values for $\mathrm{O}_{2}$ uptake are presented in $\mathrm{nmol} \mathrm{O} \times \mathrm{min}^{-1} \times \mathrm{mg}^{-1}$ protein.

\section{Mitochondrial membrane potential}

The mitochondrial membrane electrical potential was measured under the same conditions as oxygen uptake using a tetraphenyl phosphonium ( $\left.\mathrm{TPP}^{+}\right)$-selective electrode, according to Kamo et al. (1979), in a final volume of $2.8 \mathrm{ml}$. The membrane potential was calculated by assuming that the $\mathrm{TPP}^{+}$distribution between mitochondria and the medium followed the Nernst equation. For calculation of membrane potential values, the matrix volume of insect mitochondria was assumed to be $2.0 \mu \mathrm{l} \times \mathrm{mg}^{-1}$ protein.

\section{Superoxide anion production}

Levels of $\mathrm{O}_{2}{ }^{-}$release from mitochondria were determined by measuring the rate of nitroblue tetrazolium (NBT) reduction by isolated mitochondria. Nitroblue tetrazolium was added at a final concentration of $0.07 \mathrm{mg} \times \mathrm{ml}^{-1}$ and its reduction rate was measured at $560 \mathrm{~nm}$ (UV 1602, Shimadzu). Mitochondria (0.6 mg) were incubated in $1.2 \mathrm{ml}$ of incubation medium in the presence of $10 \mathrm{mM}$ succinate (fat body) and malate + pyruvate (muscle), which were added after NBT addition. Mitochondria were incubated in the presence or absence of palmitic acid (PA) and/or GTP. 


\section{UCP immunoblotting}

Cockroach fat body and muscle mitochondria were resuspended in $100 \mathrm{mM} \mathrm{Na}_{2} \mathrm{CO}_{3}(\mathrm{pH}$ 11.5) and centrifuged at $18,000 \mathrm{~g}$ for $10 \mathrm{~min}$. The supernatant containing extracted soluble and peripheral membrane proteins was discarded. The pellet was resuspended in sample buffer containing $1 \%(w / v)$ SDS, $0.1 \mathrm{M}$ Tris-HCl ( $\mathrm{pH}$ 6.8), 0.005\% (w/v) bromophenol blue and $0.5 \% \quad \beta$-mercaptoethanol and boiled for $5 \mathrm{~min}$. Purified mitochondrial membrane protein fractions (75 and $100 \mu \mathrm{g}$ ) were loaded on the gel. Electrophoresis (SDS-PAGE) was performed using a 5\% polyacrylamide stacking gel and a $14 \%$ polyacrylamide resolving gel. Separated mitochondrial proteins were then transferred onto nitrocellulose membrane. Blots were blocked with 5\% BSA in Tris-buffered saline with $0.1 \%$ Tween (TBS-T) for $60 \mathrm{~min}$ and probed overnight at $4{ }^{\circ} \mathrm{C}$ with antibodies raised against human UCP4 (Santa Cruz Biotechnology) and rat UCP5 proteins (Alpha Diagnostic) at a dilution of 1:1000. The blots were washed with TBS-T and incubated with goat anti-rabbit horseradish peroxidaseconjugated secondary antibodies (BioRad) at a dilution of 1:50000 for $1 \mathrm{~h}$ at room temperature. Protein bands were visualized using the Amersham ECL system and quantified digitally using the Scan Pack 3.0 program.

\section{UCP immunofluorescent detection}

Fat body and muscle tissue from $G$. coquereliana were collected, cleaned of tracheoles and connective tissues, and transferred to phosphate-buffered saline (PBS) medium containing $1 \%$ BSA. Afterwards, insect tissues were minced with scissors (in the case of muscle also homogenized with a teflon-glass homogenizer) and incubated with $0.4 \%$ collagenase $\mathrm{A}$ for $1 \mathrm{~h}$ at $32{ }^{\circ} \mathrm{C}$ (fat body) or with $0.4 \%$ collagenase $\mathrm{A}$ and $1 \%$ dispase for $1 \mathrm{~h}$ at $37{ }^{\circ} \mathrm{C}$ (muscle) in PBS buffer with gentle shaking. After incubation, tissues were centrifuged at $100 \mathrm{~g}$ (fat body) and $250 \mathrm{~g}$ (muscle) for $5 \mathrm{~min}$. After centrifugation of the digested fat bodies, the infranatant was carefully removed, and the remaining layer of cells was gently suspended in PBS medium and again centrifuged at $100 \mathrm{~g}$ for $5 \mathrm{~min}$. The final pellet of fat body cells was suspended in PBS buffer. After centrifugation of the digested muscle tissue, the precipitate was washed in PBS buffer and again centrifuged at $250 \mathrm{~g}$ for $5 \mathrm{~min}$. The final pellet was resuspended in PBS buffer. To visualize mitochondria, 200 nM MitoTracker Red (Invitrogen) was added to cell suspensions and incubated for $1 \mathrm{~h}$ at $37{ }^{\circ} \mathrm{C}$. Cells were then washed in PBS and fixed with $4 \%$ paraformaldehyde (in PBS) for $20 \mathrm{~min}$ at room temperature, seeded on poly L-lysine slides and allowed to completely dry. The cells were subsequently fixed with icecold methanol and permabilized with cold acetone for 5 min. Finally, the fixed cells were blocked in 5\% BSA at room temperature for $1 \mathrm{~h}$. Afterwards, the fixed cells were incubated overnight with antibodies raised against human UCP4 (Santa Cruz Biotechnology) at a dilution of 1:100 in PBS buffer with 5\% BSA at room temperature. The cells were then washed with PBS buffer and incubated for $1 \mathrm{~h}$ at room temperature with goat anti-rabbit $\mathrm{IgG}$ conjugated to fluorescein isothiocyanate (FITC) (Santa Cruz Biotechnology) at a dilution of 1:100. After a brief rinse, the slides were mounted with cover slips. Microscopic images were collected using a Nicon reversed fluorescence microscope.

Mass spectrometry analysis

After SDS-PAGE and Coomassie staining, gel slices containing protein bands were cut out and subjected to liquid chromatography coupled to tandem mass spectrometry (LCMS/MS). Analysis was conducted in the Laboratory of Mass Spectrometry, Institute of Biochemistry and Biophysics, Polish Academy of Sciences (Warsaw, Poland) using Electrospray Ionization-Linear Trap Quadrupole-Fourier Transform Ion Cyclotron Resonance. Data were analyzed automatically by database matching against the NCBI protein database using a Mascot Search (matrixscience.com).

\section{Results}

Immunological detection of $G$. cocquereliana UCP (GcUCP)

Previous studies have indicated the presence of two UCP isoforms in insects, UCP4 and/or UCP5 (Jezek 2002; Fridell et

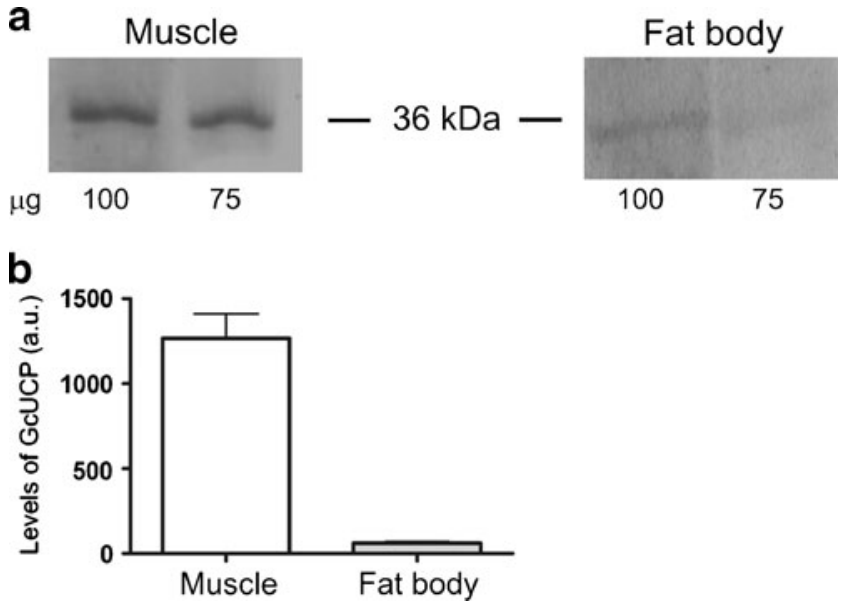

Fig. 1 Immunological identification of GcUCP protein in mitochondria isolated from muscle and fat body from $G$. coquereliana. a Primary antibodies raised against human UCP4 and rat UCP5 were used. Mitochondrial protein $(100$ or $75 \mu \mathrm{g})$ was loaded in each lane. The results are representative of at least four independent experiments. b The average UCP protein expression level $\pm \mathrm{SD}(n=6)$ in muscle and fat body mitochondria 
al. 2004; Sanchez-Blanco et al. 2006). Therefore, polyclonal antibodies raised against human UCP4 and rat UCP5 were used in total mitochondrial proteins to evaluate the presence of UCP in fat body and muscle from the cockroach $G$. coquereliana. The resultant detection was better when antiUCP4 antibodies were applied (data not shown). As shown in Fig. 1a, a single protein band with a molecular weight around $36 \mathrm{kDa}$ was detected, which indicated the cross-reaction of mammalian anti-UCP4 antibodies with the cockroach UCP homologue. GcUCP expression in cockroach muscle mitochondria was significantly higher compared to that in mitochondria isolated from fat body (Fig. 1a,b).

Using primary anti-human UCP4 antibody and secondary antibody conjugated with fluorescein isothiocyanate, we visualized the presence of GcUCP in fat body and muscle mitochondria from $G$. coquereliana. Figure $2 \mathrm{a}$ demonstrates the fluorescent detection of GcUCP in mitochondria from these cockroach tissues. The mitochondrial localization of GcUCP was confirmed by using MitoTracker Red (Fig. 2b). The merged image (Fig. 2c) clearly indicates that GcUCP is associated with mitochondria.

Effect of palmitic acid on resting respiration and membrane potential

To study the activity of GcUCP in muscle and fat body mitochondria from $G$. coquereliana, measurements were made in the presence of carboxyatractyloside, an inhibitor of the ADP/ATP antiporter that excludes its participation in free fatty acid-induced mitochondrial uncoupling. Figure 3 presents examples of oxygen consumption measurements for the resting (State 4) respiration of cockroach muscle and fat body mitochondria respiring with pyruvate plus malate (each $10 \mathrm{mM})$ and succinate $(10 \mathrm{mM})$, respectively. Increasing concentrations of palmitic acid (PA), up to $15 \mu \mathrm{M}$, were added to each type of mitochondria to reach maximum PA a
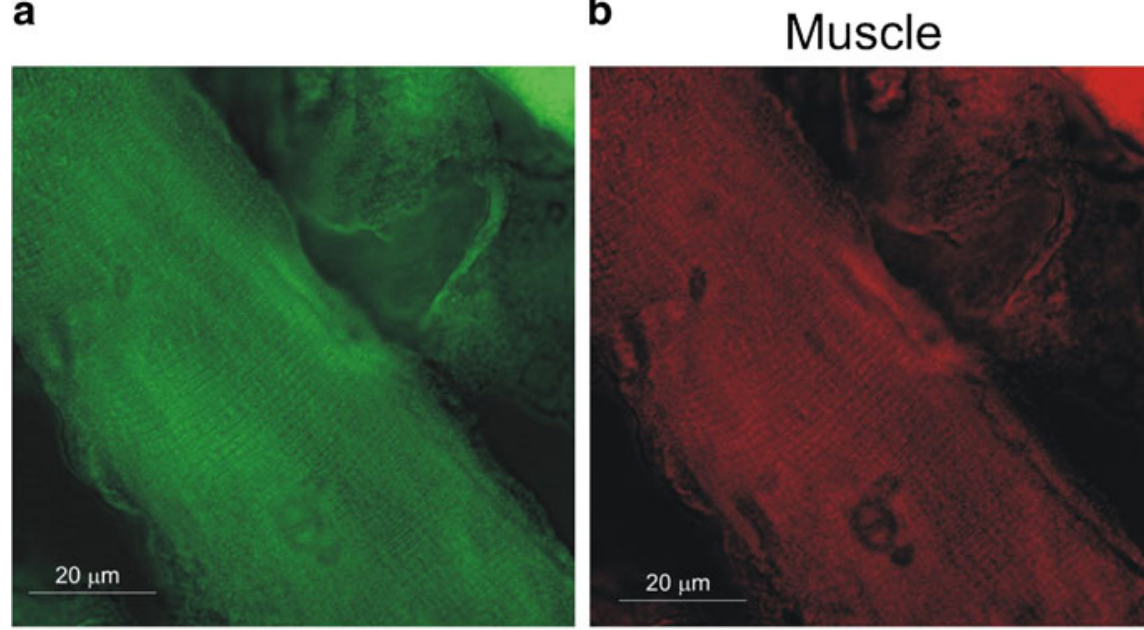

Fat body
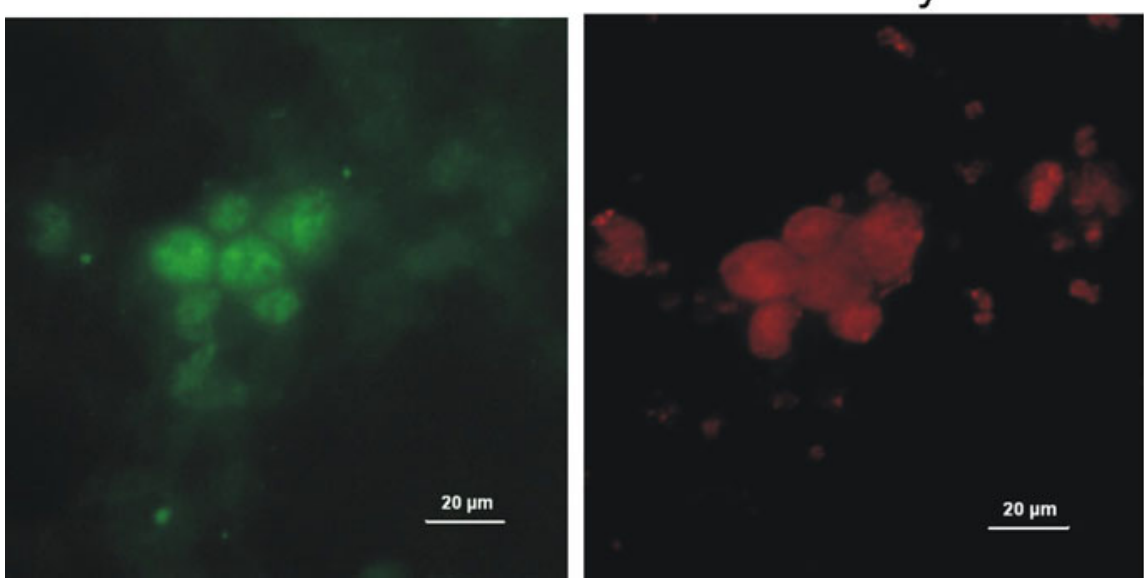

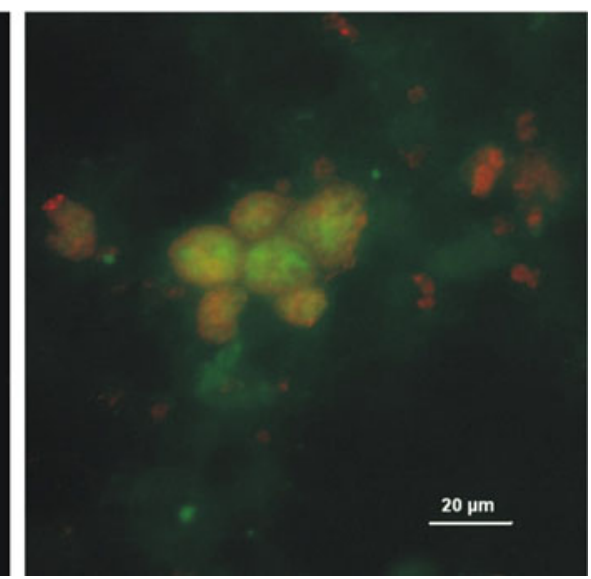

c

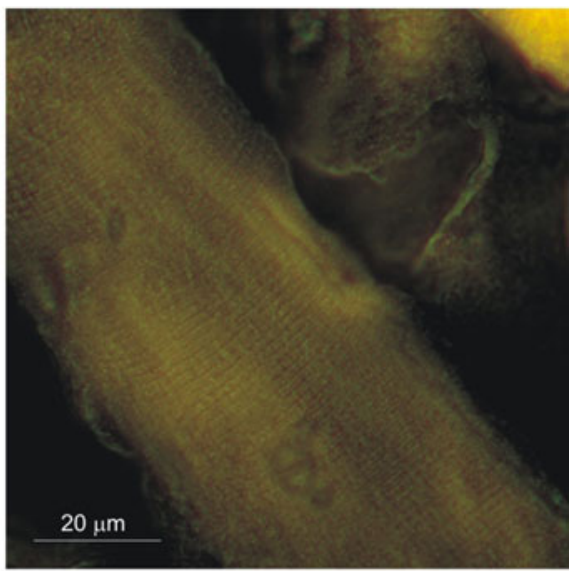

MitoTracker Red (red staining, b). The merged image (c) shows that the detected GcUCP is associated with mitochondria. The results are representative of at least six independent experiments
Fig. 2 In situ identification of GcUCP in muscle and fat body cells from $G$. coquereliana using primary anti-human UCP4 antibody and secondary goat anti-rabbit IgG conjugated to fluorescein isothiocyanate (green staining, a). The mitochondria are visualized by 


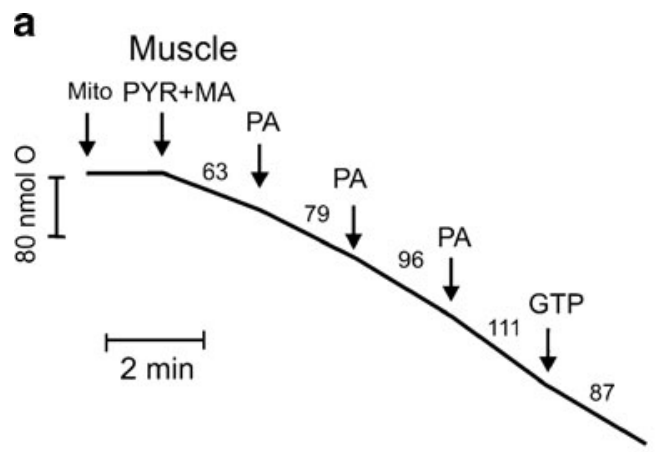

b
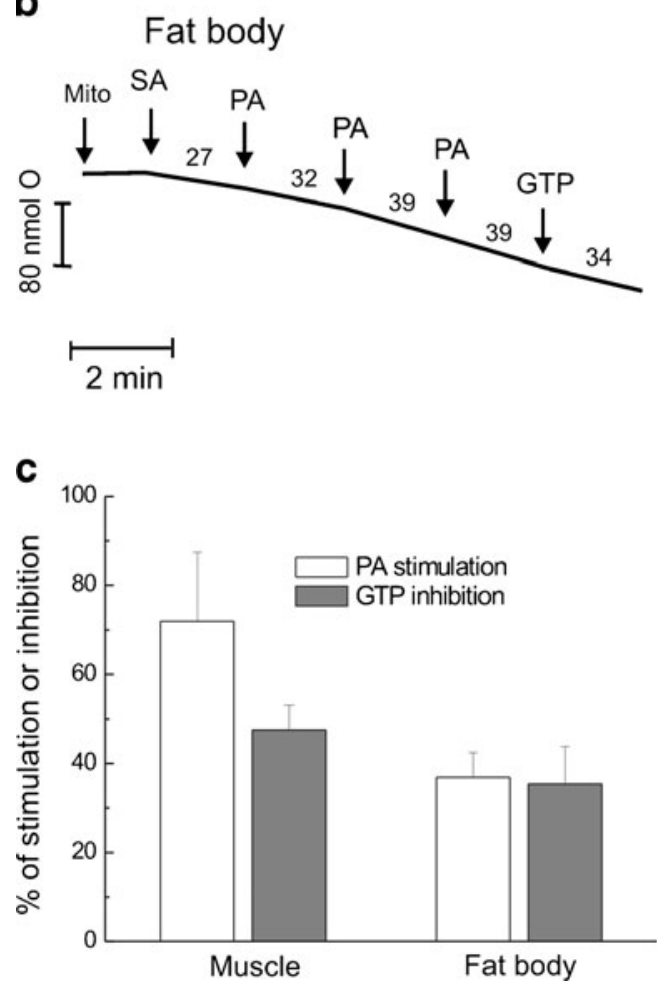

Fig. 3 Effect of PA on the respiratory rates of G. coquereliana fat body and muscle mitochondria. a Muscle mitochondria were incubated with $10 \mathrm{mM}$ pyruvate plus $10 \mathrm{mM}$ malate (PYR+MA) as oxidizable substrates. b Fat body mitochondria were incubated in the presence of $10 \mathrm{mM}$ succinate (SA) and $5 \mu \mathrm{M}$ rotenone. Measurements were performed in the presence of oligomycin $\left(1 \mathrm{mg} \times \mathrm{mg}^{-1}\right.$ mitochondrial protein) to inhibit the ATP synthase and carboxyatractyloside $(1 \mu \mathrm{M})$ to inhibit the ADP/ATP antiporter. Additions: mitochondria (Mito) $(0.4 \mathrm{mg})$, respiratory substrates, PA (5 $\mu \mathrm{M}$ each addition), and $2 \mathrm{mM}$ GTP. Examples of five measurements using mitochondria from different preparations are shown. Numbers on the traces refer to oxygen consumption rates in nmol O $\times \mathrm{min}^{-1} \times \mathrm{mg}^{-1}$ protein. $\mathbf{c}$ Percent of the maximal stimulation (mean value $\pm \mathrm{SD}, n=7$ ) of the respiratory rate by $15 \mu \mathrm{M}$ PA. Percent inhibition of the PAstimulated respiratory rate by $2 \mathrm{mM}$ GTP (mean value \pm SD, $n=7$ )

stimulation for resting respiration (Fig. 1a,b). In fat body mitochondria, after the addition of $10 \mu \mathrm{M}$ PA (which leads to maximal stimulation), respiration increased by an average $37 \pm$ $5 \%$ (SD, $n=7$ ), whereas $15 \mu \mathrm{M}$ PA stimulated the respiratory rate of muscle mitochondria by $72 \pm 15 \%$ (SD, $n=7$ ) (Fig. $3 \mathrm{c}$ ).
The addition of $2 \mathrm{mM}$ GTP partially reversed the stimulated respiration in both types of mitochondria (Fig. 3c). A slightly higher inhibition was observed in muscle mitochondria compared to fat body mitochondria $(48 \pm 6 \%$ and $36 \pm 8 \%$, $\mathrm{SD}, n=7$, respectively). However, the observed maximal stimulatory effect of PA in muscle mitochondria was significantly higher (almost doubled) compared to mitochondria isolated from fat body tissue. Similar effects were observed using another free fatty acid, linoleic acid (data not shown).

In both types of mitochondria, the stimulation of resting respiration by $10 \mu \mathrm{M}$ PA was accompanied by a decrease in membrane potential of approximately 9 and $6 \mathrm{mV}$ in muscle and fat body mitochondria, respectively (Fig. 4). The addition of $2 \mathrm{mM}$ GTP partially restored the membrane potential. The above results indicate the operation of free fatty acid-induced purine nucleotide-inhibited mitochondrial uncoupling mediated by UCP in both cockroach muscle and fat body mitochondria. Both
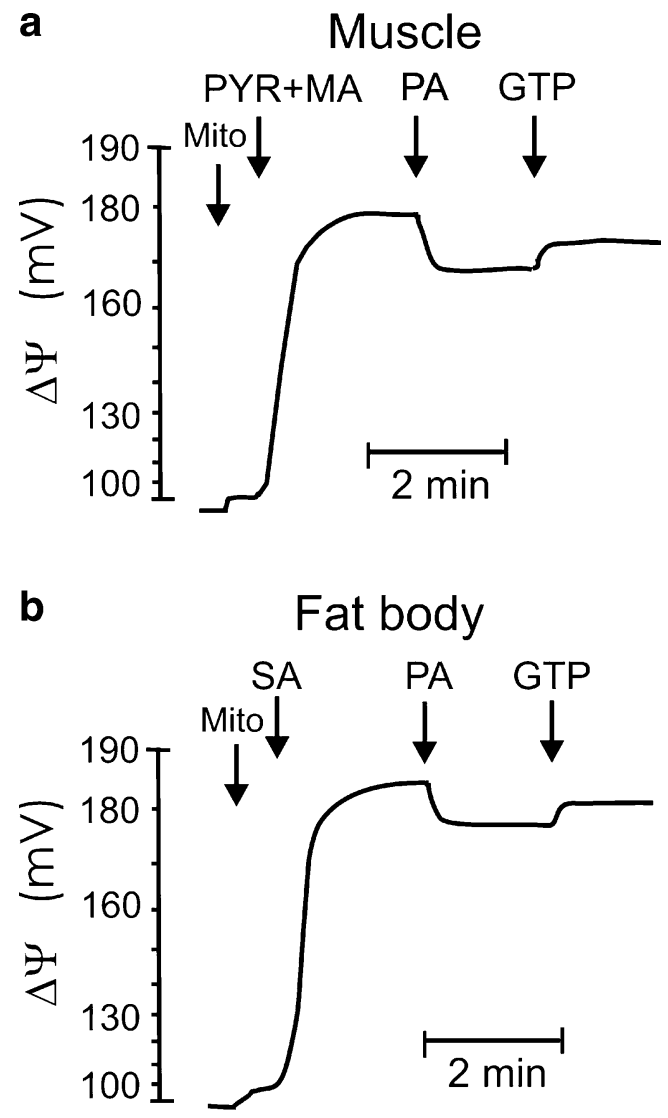

Fig. 4 Effect of PA on the membrane potential of $G$. coquereliana fat body and muscle mitochondria. a Muscle mitochondria were incubated with $10 \mathrm{mM}$ pyruvate plus $10 \mathrm{mM}$ malate (PYR+MA) as oxidizable substrates. b Fat body mitochondria were incubated in the presence of $10 \mathrm{mM}$ succinate (SA) and $5 \mu \mathrm{M}$ rotenone. Measurements were performed in the presence of oligomycin $\left(1 \mathrm{mg} \times \mathrm{mg}^{-1}\right.$ mitochondrial protein) and carboxyatractyloside $(1 \mu \mathrm{M})$. Additions: mitochondria (mito) $(1.4 \mathrm{mg})$, respiratory substrates, $10 \mu \mathrm{M}$ PA, and $2 \mathrm{mM}$ GTP. Examples of three measurements using mitochondria from different preparations are shown. Membrane potential is given in $\mathrm{mV}$ 
functional studies (Figs. 3 and 4) and immunological detection (Fig. 1) indicated a higher GcUCP protein activity and expression level in cockroach muscle mitochondria compared to fat body mitochondria.

\section{Effect of palmitic acid on phosphorylating respiration}

Phosphorylating (State 3) respiratory rates and the ADP/O ratio were measured during ADP pulses in the presence or absence of PA ( 5 and $10 \mu \mathrm{M})$ in mitochondria isolated from cockroach muscle and fat body, respectively. In both types of mitochondria, PA increased the resting (State 4) respiratory rate while it apparently did not modified phosphorylating respiration. ADP/O ratios and respiratory control ratios (RCR) were clearly lowered in the presence of PA (Table 1), which suggests the free fatty acid-induced mitochondrial uncoupling. In the presence of PA and $2 \mathrm{mM}$ GTP, the coupling parameters were partially restored. The results clearly indicate the PA-induced GTP-inhibited dissipation of the $\mathrm{H}^{+}$electrochemical gradient through GcUCP in both types of cockroach mitochondria. Despite the lower concentration (half the dosage) of PA applied to muscle mitochondria, the observed similar decrease in the oxidative phosphorylation yield (ADP/O ratios), by $75 \%$ and $72 \%$ for muscle and fat body mitochondria, respectively, indicates a higher GcUCP activity in cockroach muscle mitochondria compared to fat body mitochondria.

The effect of PA on the ADP/O ratio was concentration dependent up to $15 \mu \mathrm{M}$ (data not shown). Similar effects were observed when linoleic acid was used to activate GcUCP-mediated mitochondrial uncoupling (data not shown). The results described above indicate that free fatty acid-induced purine nucleotide-inhibited GcUCPmediated uncoupling can efficiently divert energy from

Table 1 Influence of PA and GTP on the coupling parameters. Pyruvate $(10 \mathrm{mM})$ and malate $(10 \mathrm{mM})$ or succinate $(10 \mathrm{mM})$ with $5 \mu \mathrm{M}$ rotenone were used as oxidizable substrates in muscle and fat body mitochondria, respectively. The concentrations used: $5 \mu \mathrm{M}$ (muscle mitochondria) or $10 \mu \mathrm{M}$ (fat body mitochondria) PA, $2 \mathrm{mM} \mathrm{GTP}$, and 250 nmoles of ADP (pulse) (where indicated). Mean values $( \pm \mathrm{SD}, n=4)$ for four different mitochondrial preparation are shown

\begin{tabular}{lll}
\hline & ADP/O & RCR \\
\hline Muscle & & \\
-PA -GTP & $2.27 \pm 0.07$ & $2.63 \pm 0.08$ \\
+ PA & $1.70 \pm 0.11$ & $1.53 \pm 0.13$ \\
+ PA +GTP & $2.10 \pm 0.10$ & $2.30 \pm 0.10$ \\
Fat body & & \\
-PA -GTP & $1.32 \pm 0.05$ & $3.0 \pm 0.22$ \\
+ PA & $0.95 \pm 0.08$ & $2.2 \pm 0.12$ \\
+ PA +GTP & $1.13 \pm 0.04$ & $2.4 \pm 0.09$ \\
\hline
\end{tabular}

oxidative phosphorylation in cockroach muscle and fat body mitochondria.

Effect of GcUCP activity on mitochondrial superoxide anion production

Figure 5 shows that the generation of $\mathrm{O}_{2}{ }^{-}$by cockroach muscle and fat body mitochondria was strongly reduced in the presence of PA in a concentration-dependent manner. It indicates that GcUCP activity lowers mitochondrial ROS generation in these mitochondria. The addition of $2 \mathrm{mM}$ GTP, which inhibits PA-stimulated UCP activity, increased $\mathrm{O}_{2}{ }^{--}$formation in fat body mitochondria respiring with succinate (complex II substrate). In muscle mitochondria respiring with complex I substrates (malate and pyruvate), after the addition of GTP, no effect on $\mathrm{O}_{2}{ }^{-}$production was observed.

Amino acid sequence analysis

Protein bands corresponding to an approximately $36 \mathrm{kDa}$ GcUCP band detected with anti-UCP4 antibodies (Fig. 6) were cut out from the SDS-PAGE gel and subjected to LCMS/MS analysis. The peptides were separated by liquid chromatography, sequenced and used to search databases. The genome of G. coquereliana is not sequenced and made sequence searches and comparisons more difficult. However, our amino acid sequence comparisons with known or predicted UCP isoforms clearly indicated peptide similarities in GcUCP to UCP4 isoforms. The Mascot analysis revealed four peptides found both in cockroach muscle and fat body mitochondria, and two additional peptides were found only in muscle mitochondria (Table 2). The six peptides are together composed of 55 amino acids (two amino acids are common for peptides no. 3 and 4) and likely cover around $16 \%$ of the GcUCP protein (Fig. 7). The amino acid sequences of all identified peptides were identical to at least one known or predicted UCP4 isoform in D. melanogaster, $R$. norvegicus, $H$. sapiens, $T$. castaneum, A. aegypti, P. humanus corporis, C. quinquefasciatus or $A$. pisum (Table 2). Five of the six peptides were perfectly matched to the $D$. melanogaster UCP4A isoform, which indicates a very close homology for DmUCP4A and GcUCP. The sequence comparison indicated that the cockroach GcUCP can be the closest known homologue of $D$. melanogaster UCP4A. Figure 7 shows an alignment of six of these UCP4 isoforms with peptides found in cockroach tissues marked. All matched peptides identified in cockroach $G$. coquereliana are likely parts of four transmembrane helices (except for the 1st and 6th helices). All matched cockroach GcUCP sequences contained highly conserved residues to aligned mammalian and insects UCP4 isoforms. The completely (or predominantly) conserved 


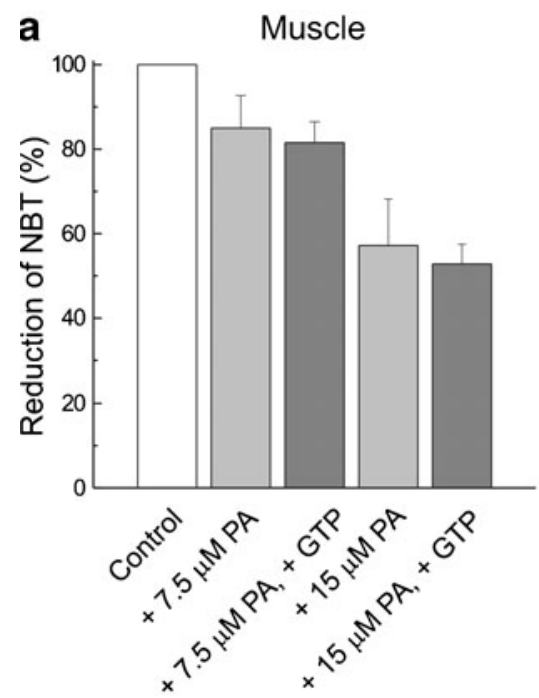

Fig. 5 The effect of a UCP activator (PA) and inhibitor (GTP) on superoxide anion generation in muscle (a) and fat body (b) mitochondria. Mitochondria $(0.6 \mathrm{mg})$ were incubated in the presence of $10 \mathrm{mM}$ pyruvate plus $10 \mathrm{mM}$ malate (muscle mitochondria) or $10 \mathrm{mM}$ succinate (fat body mitochondria) and in the presence of oligomycin $\left(1 \mathrm{mg} \times \mathrm{mg}^{-1}\right.$ mitochondrial protein) and carboxyatractiloside $(1 \mu \mathrm{M})$. Substrates were added after nitroblue tetrazolium (NBT) addition. a

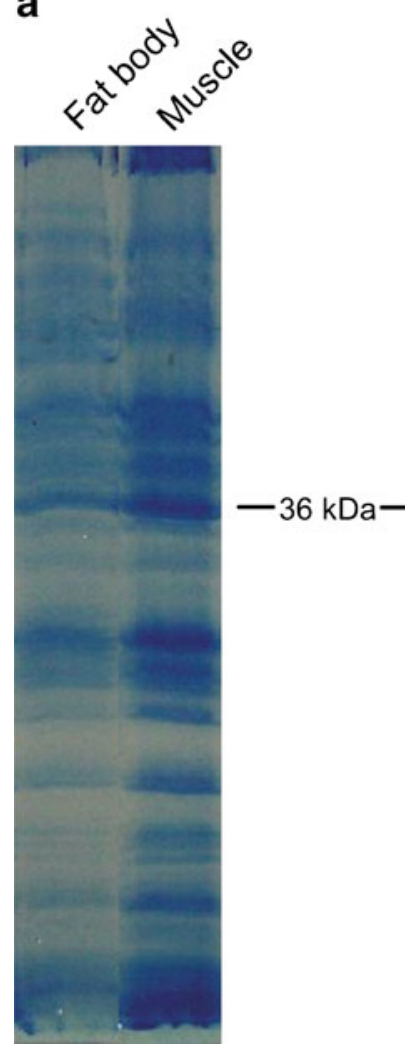

b

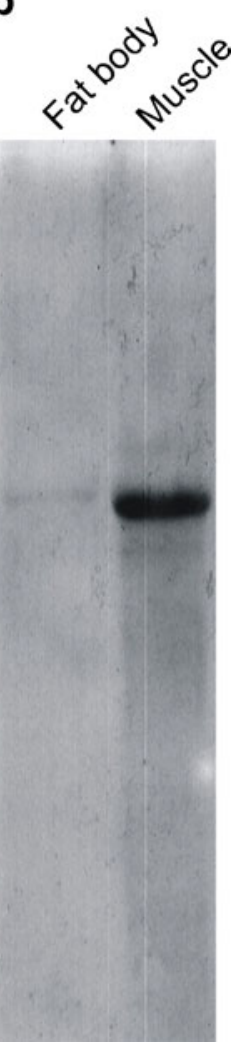

Fig. 6 a SDS-PAGE protein profile of Coomassie-stained mitochondrial proteins from cockroach fat body and muscle tissue after the extraction of peripheral proteins with sodium carbonate. b Immunodetection of GcUCP with human anti-UCP4 antibodies. The protein band (a), which corresponded to an approximately $36 \mathrm{kDa}$ band detected with the antibodies (b), was cut out of the gel and subjected to LC-MS/MS analysis

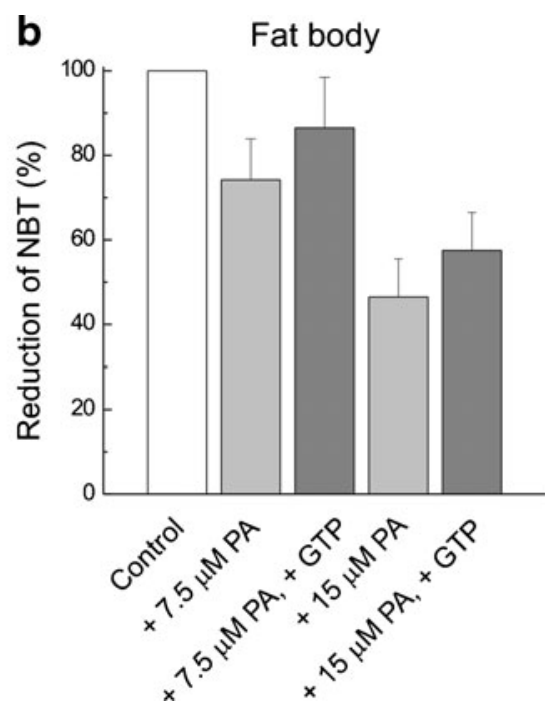

Mitochondria were incubated in the absence or presence of given concentrations of PA and with or without of $2 \mathrm{mM}$ GTP. Reduction of NBT is shown as a $\%$ of the control (no PA, no GTP). Mean values from five independent experiments are shown $( \pm \mathrm{SD}, n=5)$. For GTP effect, $p$ values were calculated. Only in fat body mitochondria, $p$ values in the presence of GTP and PA are significantly different from values in the presence of PA only ( $p<0.05$; Student's paired $t$ test)

residues of the mitochondrial carrier family signatures (Jezek 2002) were found in GcUCP peptides no. 3 and 6 (Fig. 7, red residues in black boxes). Moreover, the completely conserved residues defined as UCP signatures (Jezek 2002) were found in two cockroach peptides (no. 1 and 2) (Fig. 7, red residues in dark blue boxes). A partial amino acid sequence analysis of GcUCP protein indicates its presence in both $G$. coquereliana muscle and fat body mitochondria and its similarity to UCP4 isoforms from other animals, especially the D. melanogaster UCP4A isoform.

\section{Discussion}

This work is the first functional characterization of UCP activity from isolated insect mitochondria. Bioenergetic studies clearly indicated UCP function in mitochondria isolated from both $G$. cocquereliana leg muscle and fat body tissue, the latter being an insect analogue tissue to vertebrate adipose tissue and liver functioning as a major organ for nutrient storage and energy metabolism (Liu et al. 2009). The activity of the G. cocquereliana uncoupling protein (GcUCP) is stimulated by free fatty acids and inhibited by purine nucleotides (GTP). In resting (nonphosphorylating) cockroach mitochondria, the addition of micromolar concentrations of PA increased respiratory rates and decreased the membrane potential (Figs. 3, 4). These effects were partially reversed by $2 \mathrm{mM}$ GTP. The sensitivity of cockroach GcUCP to free fatty acids and purine nucleotides and that observed previously for $D$. 
Table 2 LC MS/MS identification of GcUCP in fat body and muscle mitochondria from $G$. coquereliana. Peptides identified in $G$. coqureliana were compared to the amino acid sequences of known or predicted UCP4s from human (hUCP4), rat (rUCP4) and some selected insects, i.e., Drosophila melanogaster (Dm), Tribolium castaneum (Tc), Culex quinquefasciatus (Cq), Pediculus humanus corporis (Phc), Acyrthosiphon pisum (Ap), and Aedes aegypyti (Aa)

\begin{tabular}{|c|c|c|c|c|c|}
\hline \multirow[t]{2}{*}{ No. } & \multirow[t]{2}{*}{ Peptides $^{\mathrm{a}}$} & \multicolumn{2}{|c|}{ Cockroach } & \multirow[t]{2}{*}{ Orthologs (the perfect match) ${ }^{\mathrm{b}}$} & \multirow[t]{2}{*}{ Score $^{\mathrm{c}}$} \\
\hline & & Fat body & Muscle & & \\
\hline 1 & K.LWQGVTPALYR.H (11 aa) & + & + & UCP4A $(D m)$ & 63 \\
\hline 2 & R.IVSYETLR.D (8 aa) & + & + & predicted UCP4 $(P h c)$, predicted UCP4A $(A p)$ & 61 \\
\hline 3 & K.VQIQMEGR.R (8 aa) & + & + & UCP4A $(D m)$, predicted UCP4 $(P h c)$ & 54 \\
\hline 4 & K.GSIPNVQR.A (8 aa) & - & + & $\mathrm{UCP} 4 \mathrm{~A}(D m)$ & 23 \\
\hline 5 & R.AALVNLGDLTT.C (11 aa) & + & + & $\begin{array}{l}\text { predicted UCP4A }(T c), \mathrm{UCP} 4(C q), \mathrm{UCP} 4 \mathrm{~A}(D m) \text {, } \\
\text { hUCP4 all isoforms, predicted UCP4 }(P h c)\end{array}$ & 114 \\
\hline 6 & R.IMNQPTDENGR.G (11 aa) & - & + & UCP4A $(D m)$ & 44 \\
\hline
\end{tabular}

${ }^{a}$ Matched peptide sequences identified in G. coqureliana

${ }^{\mathrm{b}}$ Perfectly matched (100\% identity) UCP4 orthologues of different species

${ }^{\mathrm{c}}$ Mascot search probability based Mowse score

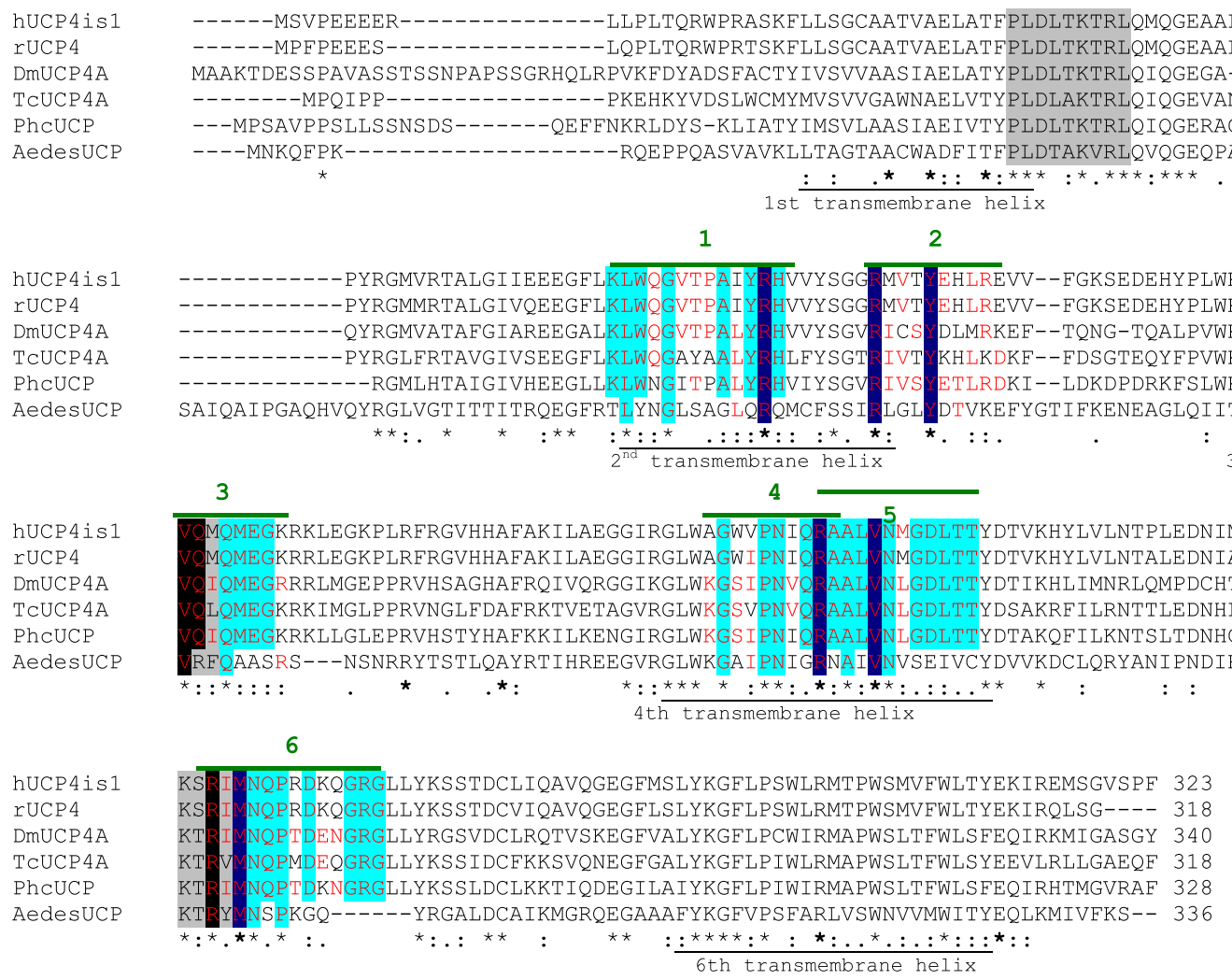

Fig. 7 Alignment of known or predicted UCP4 isoforms of human, rat and four selected insects with markings for indentified GcUCP peptides. ClustalW2 and EMBL-EBI programs were used. Peptides identified by mass spectroscopy from cockroach GcUCP are marked in red (amino acid sequence) and in green (position and number of peptides according to Table 2). Mitochondrial carrier family signatures (Jezek 2002) are shadowed in gray. The completely (or predominantly) conserved GcUCP residues found within these signatures are red in black boxes. The completely (or predominantly) conserved GcUCP residues and the other parts of aligned UCP4 isoforms (besides the mitochondrial carrier family signatures) are red in blue boxes. The completely conserved residues defined as UCP signatures (bold stars) (Jezek 2002) found in GcUCP are red in dark blue boxes. Similarity between sequences are shown as follows: * (identity), : (similarity) and . (amino acid residues belonging to the same class). Abbreviations, species and GenBank accession numbers are as follows: hUCP4is1, human $(H$. sapiens) UCP4, NP_004268.3; rUCP4, rat ( $R$. norvegicus) UCP4, NP 445952.1; DmUCP4A, Drosophila melanogaster UCP4, isoform A, NP 573246.1; predicted TcUCP4A, Tribolium castaneum UCP4, isoform A, XP_969929.1; PhcUCP, predicted Pediculus humanus corporis UCP, XP_002429177.1; AedesUCP, Aedes aegypti UCP, EAT41275.1 
melanogaster UCP5 expressed in a yeast system (Fridell et al. 2004) indicate that insect UCPs conserve this regulatory mechanism similar to mammals, plants and unicellular organisms (Fridell et al. 2004; Vercesi et al. 2006; Echtay 2007; Jarmuszkiewicz et al. 2010). In phosphorylating cockroach mitochondria, PA-induced GTP-sensitive GcUCP activity decreased the ADP/O ratio and, thus, diverted energy from oxidative phosphorylation (Table 1). Functional studies indicated a higher GcUCP-mediated uncoupling activity in cockroach muscle mitochondria compared to fat body mitochondria. This observation is consistent with the higher expression level of GcUCP observed in cockroach muscle mitochondria, as revealed by immunodetection (Fig. 1). This result may suggest an important physiological role for this protein in insect muscle tissue. On the other hand, similar to mammalian liver, the physiological role of UCP could be less essential in the insect fat body.

Using antibodies raised against human UCP4, a single band of around $36 \mathrm{kDa}$ was detected in both muscle and fat body mitochondria from G. cocquereliana (Fig. 1). Moreover, the presence of GcUCP in cockroach fat body and muscle and their colocalization with mitochondria were also visualized by reversed fluorescence microscopy using secondary antibodies conjugated to fluorescein isothiocyanate and MitoTracker Red (Fig. 2). Afterwards, the molecular characterization of GcUCP was performed by mass spectrometry and amino acid sequence analysis of peptides obtained from the $36 \mathrm{kDa}$ protein band after SDSPAGE. Amino acid sequence comparisons of the six obtained peptides (four common to both studied cockroach tissues) with known or predicted UCP isoforms clearly indicated their high similarity to UCP4 isoforms from mammals and other insects. Sequence alignments revealed that the amino acid sequences of peptides found in the cockroach GcUCP are identical to UCP4 isoforms in $D$. melanogaster (five of the six peptides matched perfectly), human, rat or other insect mitochondria. A few completely (or predominantly) conserved residues of the mitochondrial carrier family signatures and UCP signatures (Jezek 2002) were found in peptides obtained from the GcUCP (Fig. 7). Thus, our amino acid sequence analysis confirmed the existence of UCP protein (UCP4) in G. cocquereliana mitochondria and suggests a structural similarity and close molecular relationship for this protein to mammalian and other insect UCPs, especially to UCP4 isoforms. Moreover, our results support the annotation that insect UCPs appear to be more closely related to vertebrate UCP4s than to other vertebrate or invertebrate UCPs and that UCP4 might be the ancestral UCP from which other mammalian and plant UCPs diverged (Hanak and Jezek 2001; Jezek 2002).

In the present study, the existence of a functional UCP4 in the peripheral tissues of an insect is described for the first time. We have found evidence for the presence of GcUCP, an UCP4 isoform, in muscle and fat body mitochondria from G. cocquereliana. Thus, UCP4 is not only symptomatic for neural tissue, as has been described by several groups studying mammalian UCP4. The peripheral localization of UCP4 has already been observed in oocytes, liver and kidney from the frog Xenopus laevis (Keller et al. 2005). UCP4 might have evolved during evolution from peripheral tissue into a brain-specific protein. The identification of UCP4 in insect peripheral tissues (this study) supports this hypothesis.

Our results indicate that insect mitochondria, as shown for fat body and muscle mitochondria from $G$. coquereliana, can prevent ROS formation by free fatty acid-uncoupled UCP-mediated respiration. A limitation of ROS generation by UCP activity has been previously observed in plant, mammalian and protist mitochondria (Kowaltowski et al. 1998; Negre-Salvayre et al. 1997; Czarna and Jarmuszkiewicz 2005). Our data show that a PA-stimulated GcUCP activity decreases the level of superoxide anion production (Fig. 5). The inhibition of PA-induced GcUCP activity by GTP enhanced the production of $\mathrm{O}_{2}{ }^{--}$in cockroach fat body mitochondria. The addition of GTP to cockroach muscle mitochondria did not change $\mathrm{O}_{2}{ }^{--}$formation. This difference may result from the dependence of the sensitivity of free fatty induced UCP activity to purine nucleotides on Q redox state that was observed in rat skeletal muscle and brown adipose tissue mitochondria (Jarmuszkiewicz et al. 2004; Swida-Barteczka et al. 2009). However it can be concluded that GcUCP activation by free fatty acids lowers ROS formation in the cockroach both in its muscle and fat body mitochondria, which indicates that protection against mitochondrial oxidative stress may be a common physiological role of UCPs in insects.

The discovery of UCP in the cockroach G. coquereliana (UCP4 isoform) might have implications for understanding the bioenergetics, metabolism and functions of fat body and muscle in insects and could shed light on our understanding of the evolution of mitochondrial UCPs.

Acknowledgments This work was supported by the grant of the Polish Ministry of Science (N N 303291634).

Open Access This article is distributed under the terms of the Creative Commons Attribution Noncommercial License which permits any noncommercial use, distribution, and reproduction in any medium, provided the original author(s) and source are credited.

\section{References}

Borecky J, Maia IG, Arruda P (2001) Biosci Rep 21:201-212 Bradford M (1976) Anal Chem 72:248-254 
Cannon B, Shabalina IG, Kramarova TV, Petrovic N, Nedergaard N (2006) Biochim Biophys Acta 1757:449-458

Carroll AM, Haines LR, Pearson TW, Brennan C, Mbreen EP, Porter RK (2005) J Biol Chem 280:15534-15543

Czarna M, Jarmuszkiewicz W (2005) FEBS Lett 579:3136-3140

Echtay KS (2007) Free Rad Biol Med 43:1351-1372

Fridell YC, Sánchez-Blanco A, Silvia BA, Helfand SL (2004) J Bioenerg Biomembr 36:219-228

Hanak P, Jezek P (2001) FEBS Lett 495:137-141

Harper ME, Gerritis MF (2004) Curr Opin Pharmacol 4:603-607

Jarmuszkiewicz W, Navet R, Alberici LC, Douette P, Sluse-Goffart CM, Sluse FE, Vercesi AE (2004) J Bioenerg Biomembr 36:493-502

Jarmuszkiewicz W, Woyda-Ploszczyca AM, Krzeminska N, Sluse FE (2010) Biochim Biophys Acta 1797:792-799

Jezek P (2002) Int J Biochem Cell Biol 34:1190-1206

Kamo N, Muratsugu M, Hongoh R, Kobatake Y (1979) J Membr Biol 49:105-121

Keller PA, Lehr L, Giacobino JP, Charnay Y, Assimacopoulos-Jeannet F, Giovannini N (2005) Physiol Genomics 22:339-345

Kim-Han JS, Dugan LL (2005) Antioxid Redox Signal 7:11731181

Kim-Han JS, Reichert SA, Quick KL, Dugan LL (2001) J Neurochem 79:658-668

Kowaltowski AJ, Costa AD, Vercesi AE (1998) FEBS Lett 425:213216

Ledesma A, Garcia de Lacoba M, Rial E (2002) Genome Biol 3 (12):3015.1-3015.9

Liu Y, Liu H, Wang S, Jiang RJ, Li S (2009) Arch Insect Biochem Physiol 71:16-30
Mao W, Yu XX, Zhong A, Li W, Brush J, Sherwood SW, Adams SH, Pan G (1999) FEBS Lett 443(3):326-330

Nedergaard J, Ricquier D, Kozak LP (2005) EMBO Rep 6:917-921

Negre-Salvayre A, Hirtz C, Carrera G, Cazenave R, Troly M, Salvayre R, Penicaud L, Casteilla L (1997) FASEB J 11:809-815

Nicholls DG (2006) Biochim Biophys Acta 1757:459-466

Nicholls DG, Locke RM (1984) Physiol Rev 64:1-64

Sanchez-Blanco A, Fridell YW, Helfand SL (2006) Genetics 172:1699-1710

Sanchis D, Fleury C, Chomiki N, Goubern M, Huang Q, Neverova M, Grégoire F, Easlick J, Raimbault S, Lévi-Meyrueis C, Miroux B, Collins S, Seldin M, Richard D, Warden C, Bouillaud F, Ricquier D (1998) J Biol Chem 273(51):34611-34615

Skulachev VP (1996) Q Rev Biophys 29:169-202

Sluse FE, Jarmuszkiewicz W, Navet R, Douette P, Mathy G, Sluse-Goffart CM (2006) Biochim Biophys Acta 1757:480-485

Smorodchenko A, Rupprecht A, Sarilova I, Ninnemann O, Brauer AU, Franke K, Schumacher S, Techritz S, Nitsch R, Schuelke M, Pohl E (2009) Biochim Biophys Acta 1788:2309-2319

Sokolova IM, Sokolov EP (2005) FEBS Lett 579:313-317

Sujak P (1984) Oxidative Metabolism in the Fat Body during the Life Cycle of Insects, Adam Mickiewicz University Press, Seria Biologia 30, Poznan

Swida-Barteczka A, Woyda-Ploszczyca A, Sluse FE, Jarmuszkiewicz W (2009) Biochem J 424:297-306

Vercesi AE, Borecky J, Maia ID, Arruda P, Cuccovia IM, Chaimovich H (2006) Annu Rev Plant Biol 57:383-404

Yu XX, Mao W, Zhong A, Schow P, Brush J, Sherwood SW, Adams SH, Pan G (2000) FASEB J 14:1611-1618 